\title{
Meaning and Practice of Palliative Care for Hospitalized Older Adults with Life Limiting Illnesses
}

\author{
Bethel Ann Powers, ${ }^{1}$ Sally A. Norton, ${ }^{1}$ Madeline H. Schmitt, ${ }^{1}$ Timothy E. Quill, ${ }^{2}$ \\ and Maureen Metzger ${ }^{1}$ \\ ${ }^{1}$ School of Nursing, University of Rochester, 601 Elmwood Avenue, Box SON, Rochester, NY 14642, USA \\ ${ }^{2}$ School of Medicine and Dentistry, University of Rochester, 601 Elmwood Avenue, Box SON, Rochester, NY 14642, USA \\ Correspondence should be addressed to Bethel Ann Powers, bethel_powers@urmc.rochester.edu
}

Received 26 October 2010; Accepted 22 January 2011

Academic Editor: Nancy Hodgson

Copyright (C) 2011 Bethel Ann Powers et al. This is an open access article distributed under the Creative Commons Attribution License, which permits unrestricted use, distribution, and reproduction in any medium, provided the original work is properly cited.

\begin{abstract}
Objective. To illustrate distinctions and intersections of palliative care (PC) and end-of-life (EOL) services through examples from case-centered data of older adults cared for during a four-year ethnographic study of an acute care hospital palliative care consultation service. Methods. Qualitative narrative and thematic analysis. Results. Description of four practice paradigms (EOL transitions, prognostic uncertainty, discharge planning, and patient/family values and preferences) and identification of the underlying structure and communication patterns of PC consultation services common to them. Conclusions. Consistent with reports by other researchers, study data support the need to move beyond equating PC with hospice or EOL care and the notion that EOL is a well-demarcated period of time before death. If professional health care providers assume that PC services are limited to assisting with and helping patients and families prepare for dying, they miss opportunities to provide care considered important to older individuals confronting life-limiting illnesses.
\end{abstract}

\section{Introduction}

The fields of palliative and end-of-life care are plagued by semantic confusion. The confusion has resulted in conflation of these concepts around concerns about death and dying that limit understanding of their distinct and synergistic properties. This paper illustrates the distinctions between as well as the intersections of palliative care (PC) and services exclusively devoted to end-of-life (EOL) care, such as hospice. The illustrations are from case-centered data of older adults cared for during a four-year ethnographic study of an acute care hospital PC consultation service.

We do not view institutionally based PC and hospice services as synonymous. PC services may be provided at any time along the illness trajectory and may be delivered at the same time as curative treatments. In contrast, organized hospice services generally involve provision of PC focused exclusively on comfort care of persons identified as having a short life expectancy (usually six months or less) and who are no longer seeking disease-directed treatments. To be enrolled in hospice, patients and families must make the difficult transition to accepting that they are entering the end stage of life and that medical treatments to prolong life are no longer feasible. PC services include managing pain and other physical symptoms, improving quality of life, providing psychosocial, emotional, and spiritual support, dealing with uncertainty about treatment options and goals of care, and, through communication and coordination, simplifying navigation through the health care system.

In this paper we argue for the importance of a broad conceptualization of PC services for hospitalized older adults and their families who are dealing with uncertainties, transitions, and other changes within the course of life limiting illnesses. We further argue that every person's experience is unique and, thus, views on "how to live with dying" pose different challenges for and among dying persons, their families, and members of the professional teams that care for them in acute care hospital settings. The research results described reflect some of those challenges. 


\section{Methods}

2.1. Study Design and Sample. The data analyzed for this report are from a broader ethnographic examination of how a PC consultation service and other clinical services work together within the culture of a 750-bed academic medical center. The analysis is based on a subset of that study's case material concerning older adult patients, ages 65 and above $(N=11)$. The mean age of patients in this subsample was 81 years. Data accumulated for the 11 cases consists of 44 transcripts of in-depth individual interviews and associated fieldnote observations. All were cases of seriously ill patients with diagnoses that included advanced metastatic cancer, stroke, heart failure, intracranial hemorrhage, progressive ALS, ruptured aneurysm, burns, spinal fracture, and COPD.

In this paper, demographic and other personal details derived from cases are minimized to protect the privacy of individuals and avoid unnecessary individual tags that could threaten participants' anonymity [1]. Researchers obtained signed informed consent for all participants and the research was approved and overseen by the Research Subjects Review Board of the sponsoring university.

2.2. Data Collection. In this study, we were able to observe how PC team members actively cooperated and collaborated in patient care alongside members of a variety of medical and surgical referring teams. The PC team was observed throughout the day and into the evening on weekdays and some weekends over a 30 -month period. A series of case-based in-depth interviews with patients, families, and individual members of both the PC and referring team was phased in over a period of 21 months. Cases $(N=$ 27 ), as the unit of analysis, were purposefully selected to achieve variation in makeup of the referring team and location of in-house service, reasons for the PC consultation, and patient/family demographics (e.g., age, sex, ethnicity, diagnosis). Interview guide content fields included reasons for the PC consultation, goals of care, values and preferences associated with decision making about treatment and goals of care, expectations and perceptions of PC and opinions about the outcomes of PC involvement.

2.3. Data Analysis. Analysis of the 11 target cases (out of 27 cases-40\%) involving older adults focused on types of PC services provided in response to requests for consultation. It consisted of (a) locating each case's basic plot or storyline as viewed by the different narrators of the experience (patients, families, clinicians, researchers), (b) identifying actions taken by the PC team and reported responses to them, (c) classifying cases according to dominant plotline/storyline features, and (d) identifying common themes in order to provide an integrated portrayal of the practice and meaning of PC for this vulnerable population.

\section{Results}

We begin with four practice paradigms-EOL transitions, prognostic uncertainty, discharge planning, and patient/family values and preferences - that illustrate what we learned about PC services from observations and interviews with study participants. The four paradigms are composites of individual cases and exemplify clinical situations that involved requests for PC services. Two cases served as information sources for more than one paradigm. (Each case consists of interviews with patients and families, a referring team member, and a PC team member.) We then identify themes common to all of the examples followed by our conclusions about these results in relation to relevant literature.

\subsection{PC Consultation Services: Practice Paradigms}

3.1.1. End of Life Transitions. Description to illustrate this paradigm draws upon four cases where patients were near the EOL and the PC team's expertise related to patient comfort and environmental support was a key element of consultation requests. The following quote is an example of a supportive effect:

[Spouse]: "You don't always know the right questions to ask to get what you need... some of the information is confusing... I'm glad the referral was made because it is a very comforting feeling to know that you're not going through it and doing what we have to do alone."

Amidst the certainty of impending death, inherent uncertainty about when death will occur and how dying persons will live until they die can be especially troubling. Patients and families often described receiving both too much and too little information. For example, a family member said: "To make decisions, we need to know the truth up front.... We came to the hospital with [one] group [patient's primary team]; and that [team] changed after we got here; then we came to ICU on Sunday, when things [patient circumstances and teams] changed again; and Monday, Dr. X was the one that took over; and Dr. Y was the one we had before; but then Dr. Z said to call him (we met him just briefly) and he said he'd be back, but he was off yesterday, then things happened...; we have met so many; I'm sorry I cannot remember their names... so we're feeling pretty good about the PC group because that's the same person we're talking to all the time. That really does make a difference." The PC team provided continuity and accompaniment as the patient was getting sicker, potentially approaching the EOL. They helped families obtain information on which to base EOL care decisions in a form that they could understand, inviting them to ask questions and raise concerns. The kinds of questions raised included: Is it time to transition to a hospice philosophy of care, and if so, should it be provided at home, in-hospital, or freestanding hospice facility? How will patient comfort be addressed? How can we [families] maximize the quality of time spent together? When extubation is involved: What are the criteria and protocols? What will be done for the patient? How can family members prepare and care for one another?

The PC team was sometimes consulted before the patient had clearly transitioned to an EOL situation both 
to manage symptoms and to establish a relationship in case the patient's clinical situation deteriorated. For example, pain and symptom management were frequent reasons for requesting a PC team consultation. The following quote represents a typical concern.

[Referring team member]: "We had a bit of a struggle as far as pain management went because she went through some episodes of delirium... and we were trying not to exacerbate it and to be very lenient on narcotics even though she was in pain."

Referring teams appreciated the expertise as well as the extra time extended by team members to individualize symptom relieving 'recipes' that more closely met patient needs. These types of contacts with the PC team, for some patients, became entrees for a more extended relationship. A physician explained: "Sometimes the families are a little bit reluctant [because they associate PC with EOL and hospice] ... And if we do have trouble [where] we feel that PC could be helpful, we tend to use that [management of pain or other symptoms] as a lead-in to get the family introduced to the folks who are on the PC team and develop a relationship. And then things sort of morph... [i.e.] the role of the PC team with that patient tends to change over time."

Chronically and seriously ill older adults and their families may become used to accepting and adjusting to the changes that occur with multiple life threatening episodes and remissions, with good times and bad times. Experience can make it hard to recognize endings to repeated roller coaster illness cycles. Thus, patients, families, and clinicians may not be arriving at recognition of an adverse event and/or approaching EOL with similar understandings and at a similar pace, as illustrated by the following quote.

\begin{abstract}
[Referring team member]: "Palliative care was consulted to help improve communication and help the family deal with everything that was going on.... The palliative care team serves sometimes as a buffer for members of the [referring] health care team.... So they're walking us through the process as much as we're walking with the family through the process."
\end{abstract}

In such situations, PC team consultation on goals of care provided bidirectional interpretations of patient/family and clinician perspectives regarding how the dying process, with its acknowledged attendant uncertainties, should be managed. A family member expressed the need for PC team intervention as: "A lot of us weren't on the same page [and] it led to a lot of miscommunication between family members and the team of doctors that [were] working with us." A common response from clinicians was "... oftentimes these are difficult discussions with the family talking about very sensitive and emotional issues... PC team people are experienced in... keeping everyone focused on doing what the patient would want at that time."

Location of death, as illustrated by the following quote, was a frequent concern, given that hospice care can be delivered wherever persons reside (institution, home, or hospice facility).

[Spouse] "Our son promised, 'We're going to get you home, Dad.' We know it may not happen, but we want to try for it because that's still what he [the patient] would like."

In the US, older adults with life limiting illnesses are more likely to die in a hospital than to die elsewhere [2], but it was not surprising that participants in this study expressed strong desires to be at home or to bring loved ones home to die. Helping families envision what a supportive environment for the patient would look like and striving to best accommodate patient/family needs required individualized plans responsive to patient condition and family/community resources. Universally voiced worries about symptom control for the patient, especially pain control, were not the PC team's only focus. A family's dynamics are deeply affected by the transition toward death of one of its members, which is one of the reasons the discussion about hospice is so challenging. Patient and family tendencies to perceive initiated discussions about hospice as 'bad news' often pose barriers to timely provision of its unique benefits. However, these barriers can be overcome "by considering indicators of a limited prognosis, framing the hospice discussion in terms of the patient's goals and needs for care, and recommending hospice when [physicians] think it is the best option (p. 447) [3]." Spending time to learn and understand family history and relationships, as well as possible, was a significant aspect of tailoring PC interventions to the unified needs of patients and families.

3.1.2. Prognostic Uncertainty. Description for this practice paradigm draws on two cases involving the challenges attendant to prognostic uncertainty when probable future outcomes of patients' conditions cannot be immediately ascertained.

\section{[PC team member]: "It's always the case with a stroke patient... you don't know for sure... time was the issue.... How long do you keep doing things that the patient wouldn't like done?"}

Prognostic uncertainty is a prevalent reality in the care of seriously ill patients. For example, it is not always clear if a patient's living will rejection of resuscitation or invasive procedures, such as IVs and feeding tubes, should be honored in emergency situations where some degree of recovery may be possible. But if, in time, the benefits of intervention fail to materialize, the possibility of withdrawing treatment becomes a difficult, emotion-laden decision, falling frequently to family surrogates. Services of the PC team in these circumstances were directed toward helping decision makers clarify their values and concerns in light of patients' values, expressed preferences, and current conditions. Family intellectual and emotional struggles are eased by the foresight afforded by advance directives/living wills but the anxiety and sorrow of determining when is the right time to follow these choices and what the 
implications of following them will be must be lived through. For example, families who were unfamiliar with the dying process often expected death to quickly follow a decision to discontinue invasive treatments. "Where I'm having difficulty," said one family member, "is [my] knowing what Mom would want and trying to separate [that from] what's my comfort zone... what's best for me.... She said she didn't want to be kept alive... so I don't understand why she's still here." When patients do not die immediately, families often question if their decision to stop invasive treatments was the right thing to do. "It's very hard," a PC team member said. "I assembled the whole team and we explained the physiology of death from that condition. She was so relieved."

There is no set-in-stone formula for prognosticating in cases involving injuries such as damage resulting from a stroke. This type of situation gives rise to many questions and concerns.

[Family Member] "All of a sudden he's taking
[nourishment]...I'm perplexed about the next
step.... Is the outcome going to be so different? Is
he going to be able to participate in therapy? Is his
paralysis going to be gone? Will he have speech?
... of course, nobody can know."

In the absence of certainty, clinicians may voice various opinions over time, based on the clinical evidence at hand. But systematic symptomatic management while waiting for a clearer picture of a patient's prognosis to emerge may, from a family's perspective, also feel like uncoordinated care. Coming to terms with uncertainty about the future is challenging. In these cases, the PC team's filtering of disparate information to make it understandable and the team's orientation toward 'Hope for the best; plan for the worst' tempered family responses to the tentativeness of patient outcomes that they faced. As the two patients examined here had clinical courses that diverged, decision support focused on interpretations of each patient's advance directives, involving for one, the point for letting go and for the other, rekindled hope for improvement in view of subtle changes: "They [family members] feel supported, no matter," said a referring team member. "And they know, at any time, they can reverse what they might want to do for him [hospice] if he starts to improve. So it's not like once you've started in this route that he's got to stay in that."

3.1.3. Discharge Planning. Description for this practice paradigm draws on two cases involving the collaborative, interdisciplinary, decision making activities accompanying the social intervention of hospital discharge planning. For older persons with multiple serious co-morbidities, discharge planning concerns can multiply and become increasingly complex with repeated hospitalizations. The PC team's assessment of patient circumstances was helpful in uncovering the interplay between viable options, a patient's personal choice, and actual disease state, as in the following example.
[Daughter]: "How am I ever going to translate this [Medicaid application] to him? .... [His possessions are] his legacy... what he wants to leave his children and grandchildren...."

Admission to a skilled nursing facility with or without added hospice support may be the reasonable option for persons unable to manage living safely at home with what family, friends, and/or home care services can provide. That choice for many, however, requires divestiture of personal assets. Alternatively, a patient's choice of home hospice enables retention of home and property, but hospice agencies cannot provide coverage for patient disease states that require 24-hour care. "I definitely think [the PC team] made a difference," a referring team social worker said about a patient's change in attitude about the prospects of choosing comfort and safety over "struggling and being uncomfortable [at home]." She added, "I also think it was helpful to his daughter to hear what the team was thinking as well as what he [the patient] was thinking, because I don't know that they had really discussed some of those things before."

In the fragmented US health care delivery system, poorly executed hospital discharge plans have serious consequences for medically fragile individuals with continuous and complex care needs [4-6]. Patients and families were driven by fears and reluctance to face the personal, social, and financial fallouts of nursing homes as discharge destinations and by desires for a return to a sense of normalcy and freedom that returning home symbolizes, for example:

[Spouse] "It looked like they were pushing me to send him to [a nursing home] and I just wasn't pleased with the care he might have gotten there... We're looking to take him home."

The discharge planning experience often pitted the needs, competing demands, and desires of patients and families against limitations (e.g., financial, time, geographic, and type of insurance coverage) of both personal and communitybased resources. With other health professionals, PC team decision support focused on helping reduce emotional barriers to patient/family hard life choices while simultaneously helping other involved health professionals understand what quality of life looks like from a patient/family perspective. These interventions paved the way for coordinated action plans with fallback options that focused on reconciling patient/family preferences and available resources with considerations for patient comfort and safety.

3.1.4. Patient/Family Values and Preferences. Description draws on five cases where patient and family values and preferences came to the forefront of evaluation and/or mediation by the PC team. Help in understanding and applying patients' previously expressed treatment preferences to current situations was sometimes needed. When patients were able to be involved, the PC team took care to evaluate their understanding of the consequences of their preferences. They helped patients and families determine if the current situation matched what was earlier envisioned by the patient; 
and they checked for doubts, changes of heart, or needs for a more nuanced approach, for example:

[PC team member]: "When we talked to the patient we realized that it wasn't really just a comfort care approach and that he still wanted to be on antibiotics and still give himself a shot, so we kept him on ... like a trial to see how he went. It was kind of like sink or swim."

In instances where a referring team presumed that the PC team would help them direct patients and families toward limitation of disease-driven treatment and acceptance of EOL care, conversations, such as the following, about moving toward or supporting a different outcome could take them by surprise.

[PC team member]: "Our understanding was that his life expectancy was a matter of weeks and it seemed reasonable that a hospice level of care would be appropriate.... But he seemed to be good; he was not declining; and his wife wanted maximal supportive care. It took persistence to make it very clear to the [referring] team that she wanted this maximal supportive care, did not want a hospice level of care, was well within her rights to ask for that level of care, and that they were going to have to deal with that."

Respectful discussions about differing opinions on goals of care can be uncomfortable. In such circumstances, a patientcentered orientation helped PC team members better understand what might undergird ideologies guiding professional judgments of referring teams and the life experiences and values of patients and families, always with the intent to prevent and relieve suffering. This at times enabled them to see possibilities for judicious compromises that could ease a family's suffering without discomfort to the patient. A PC team member explained, "It's key to be flexible and roll with the clinical scenario. It was clear that she [the wife] was not comfortable [and] he's [the patient's] not any worse. If anything, he might be a little better.... Over time, either the clinical situation changes or I learn more about the dynamic... what the patient's and family's needs are."

Persons who reach old age with serious and eventually fatal chronic conditions may not be recognizably at EOL, and may not meet hospice criteria according to prognosis even if they want a purely comfort-oriented approach to treatment. Dying trajectories can be unpredictably short or prolonged, and often a mix of curative and palliative treatment is needed to manage both disease and symptoms.

[PC team member]: "If I were to guess why we were asked to see her... [it] was because she was elderly and people are saying, 'Geez... we don't want to do any harm here' and 'What should we be doing?' I mean, she was $95 \ldots$ [but] she was a really good 95."

In cases where there are doubts about a patient's ability to tolerate presumably negligible benefits of disease modifying regimens, referring teams may expect a $\mathrm{PC}$ consultation to result in a patient decision to reduce reliance on diseasedriven treatment. However, in all cases, the starting point for the PC team is patient/family wishes and preferences. The work of exploring reasonable options, in the context of best available information regarding prognosis, and clarifying tradeoffs involved in choosing one decision pathway over another begins there. For example: "There was a question about whether she should go to hospice care," said a PC team member, about this patient whose disease status foretold a future of constant monitoring and repeat hospitalizations. "But she clearly would love to keep living a lot longer, was willing to take medicine to do that and be monitored... she had [a strong support system]... and also the treatment [optimization of her heart medication regimen] was likely to be helpful to her.... So everyone was moving in the same direction... once they thought it through."

3.2. Common Themes. The above categories of PC services linked to the primary plotlines/storylines in this sub-sample of 11 patient-focused cases describe what is in the dataset. Themes identify meaningful repetitive refrains that run across informational groupings and all through the data [7]. Here, they are important for understanding the underlying structure of PC consultation services and for making sense of the communication patterns that requests for consultation engender. In these data there were two clear themes related to PC services provided in response to requests for consultation.

Structure/Clinical Expertise Guided by Goals of Care. Demographic changes in the US, resulting from improved public health and medical treatments, have translated into this century's older adults living longer and for longer periods of time, 2 years on average, from the onset of serious illness to death [2]. This is a stressful period for ill individuals and their families, replete with multiple and sometimes conflicting goals of care (e.g., remission or cure, maintenance of function and independence, relief of suffering, prolongation of life, quality of life, a 'good death') that change and fluctuate with the vicissitudes of the shared illness experience. In this study, regardless of the reason for a PC referral, the progress and outcomes of the consultation were influenced by the orientations of referring teams and patients/families toward goals of care. Consensus building surrounding goals of care ensured that PC team members' expert clinical skills related to relief of physical pain and discomfort could be better utilized and extended, collaboratively, to relief of other forms of human suffering. Extended and/or unpredictable illness trajectories involve many turning points. In accordance with some understanding of goals of care and with patient safety and comfort as prime considerations, the point of the journey at which patients were encountered by the PC team influenced direction of its clinical services toward life sustaining treatment, comfort care, or a combination of both types of measures.

Communication Patterns/Decision Support. Some understanding of goals of care was critical to PC team offerings 
of decision support to patients, families, and referring teams. Communication patterns of PC team members focused on combining diagnosis and delivery of expert comfort care measures with the integration of clinical perspectives and patient/family experiences. This work required, in varying degrees, time, patience, and diplomacy. Armed with insights gained from careful and continuous data gathering, team members spent time with families helping them to interpret the meaning of clinical signs and difficult to understand and/or absorb information. Alert to potential conflict among family members or between families and referring teams, they arranged meetings to enable families, clinical team members, and relevant others, such as clergy or ethics consultation services, to engage in clarifying and resolving patient-centered concerns.

Decision support involved efforts to: (a) frame the decision and available options in a balanced way, (b) explain known and potential risks and benefits of patients' options, based on experience and the best available evidence, and (c) provide alternate ways of thinking about the specific patient/family situation that might lead to different choices and outcomes. The previously presented practice paradigms exemplify situations that invited different types of decision support, which in turn required an understanding of existing viewpoints about goals of care among patients, families, referring, and PC clinical teams.

Patient/family satisfaction was consistently linked to PC team members' rapport building, psychosocial exchange, and patient-centeredness skills. Typical comments were: (a) "I had a sense that they weren't looking at the clock. They really listened and the time they spent was really for him [the patient]." (b) "They can explain things [like] what you might be facing [and] they weren't hard to reach. They were available and they gave me comfort." (c) "They make sure that we're all comfortable with everybody making the decisions as a group now."

Referring team satisfaction was consistently linked to PC team members' partnering skills that demonstrated understanding of the clinical picture and facilitated their goals and objectives in the patients' interests. Typical comments were: (a) "Palliative care helps soften the blow of the final outcome. That's what they're educated to do [facilitate EOL discussion]. They open up that line of communication for us when it's a touchy situation." (b) "[The patient] had a long history of chronic pain issues, so our goal was to make her comfortable." (c) "Where individuals are not certain about which avenues to take, it [PC consultation] makes sense. It's more than just end-of-life care. It means having the patient come to a better understanding of his or her illness and what the options are, and facilitating those [feasible and desired outcomes] as best as possible."

Key to PC team consultations was successful collaboration with referring teams and families that maximized and directed everyone's energy toward the best possible outcomes for patients. Team members' specialized contributions involved expertise in communication, in the context of poor or uncertain prognoses; pain and symptom management; advanced knowledge of drug use, dosage, and side effects; EOL care; and patient/family support.

\section{Discussion}

The analysis demonstrates a broad spectrum of institutional PC services, which included but was not limited to pure palliation and referral to hospice when patients were seen to be approaching EOL. The distinctiveness of PC and hospice EOL services was most apparent when patients' goals and preferences were in the direction of regaining and sustaining a manageable lifestyle for living with advanced illness. The interweaving of PC and hospice care philosophies observed in PC clinicians' consultation experiences was synergistic, evidencing mindset flexibility that embraced supportive services to seriously ill individuals throughout the course of their illness journeys, with particular attention paid to individual patient and family values, beliefs and goals. In this regard, the overarching umbrella of PC services was compatible with the most aggressive disease-directed treatment, on the one hand, or with purely comfort-oriented treatment (hospice), on the other.

Data from our study support the need to move beyond equating PC with hospice or EOL care, and away from the notion that EOL is a well-demarcated period of time before death. This is not unique to our study, however. Recognized leaders in PC have argued eloquently for stepping away from the dichotomy of curative versus comfort care, such as that provided by hospice [8-12], and moving toward a broader conceptualization of PC services and their intended recipients. In more recent years, there has been a push to extend PC services to those with noncancer diagnoses, such as dementia [13,14] and heart failure [15-17], which disproportionately affect elders. The PC focus often is on management of symptoms which, for some chronic and terminal non-cancer conditions, may respond well to aggressive treatment. As individuals transition toward the interface of PC and organized hospice EOL care, the constraints of hospice policy can shape options that patients and families find viable. For example, whereas cessation of disease modifying treatments as a condition for enrollment in hospice often brings relief to cancer patients, the fear of exacerbation of the underlying disease makes persons with some other diagnoses unwilling to give up diseasemodifying treatments that provide symptomatic relief in order to qualify for hospice services.

The conflation of PC with hospice care has been associated with a preoccupation with prognostic certainty by clinicians, policy-makers, patients, and families. Precisely defining an illness trajectory is challenging in most life limiting illnesses or chronic conditions, but particularly so in many conditions, such as heart failure $[18,19]$ and dementia [14], which affect so many elders. Clinicians sometimes delay referrals to PC, feeling that they must correctly identify a time-limited EOL period. Similarly, many loved ones asked to make decisions for incapacitated patients, as in the case of later-stage dementia or other advanced illness, find it distressing to do so in the context of an uncertain disease trajectory $[14,20,21]$. Furthermore, many patients who would benefit from PC services refuse, feeling that they are not "actively dying" [22]. In a study by Quill and colleagues [23], 215 first-person patient responses to the 
question "What is most important for you to achieve?" asked at the time of initial PC consult were reviewed and categorized. Only $11 \%$ of the respondents indicated that preparation for dying was most important for them to achieve. If providers assume that patients and families referred for PC consultations are seeking assistance with or preparation for dying, then they miss opportunities to provide care considered important to those confronting life limiting illnesses.

Inpatient $\mathrm{PC}$ consultations have been linked with several positive outcomes, such as perceived increased quality of care $[24,25]$, decreased symptom distress [24-27], and increased patient and/or family satisfaction with care $[24,26]$. These outcomes are consistent with reports of participants in our study regarding their interactions with the PC team.

Data from our study also support the importance of acknowledging the uniqueness of each patient and family's experience, as specified by the national PC guidelines [28]. Consistent with reports by other researchers [26], in our study, patient and family satisfaction was linked to PC team members' communication and patient-centeredness skills. The PC team members were consistently focused on assisting patients and families with articulating goals of care and consensus-building around those goals of care with referring team members.

All of the patients in this study subsample were living with potentially life limiting illnesses. However, few were overtly and officially labeled as at the EOL. Given the acute care hospital context in which these older adult patients were encountered, timeframes for contracting with hospice were sometimes too short to be of use to those who were imminently dying. Indeed, often there were no clear demarcations of a patient's illness trajectory. This lack of demarcation is sometimes frustrating to clinicians and policy makers, hoping for clear and certain parameters for decision making and resource utilization. In contrast, because PC leaders have pushed heavily, both within and beyond the boundaries of the 'end-of-life timeframe', they have been able to extend much needed services to hospitalized older adults living with serious diagnoses and distressing symptoms. Such services, as described by the patient and family participants in our study, were helpful in navigating the increasingly complex era of living with advanced and ultimately life limiting illness.

\section{Acknowledgments}

NIH/NINR: 1RO1 NR008790-05 (PI: S. A. Norton) Palliative Care in the Acute Care Setting NIH/NINR: F31NRO12084 (PI: M. Metzger; Sponsor: S. A. Norton) Patients' Perceptions of the Role of Palliative Care in Late-Stage Heart Failure.

\section{References}

[1] J. M. Morse, “'What's your favorite color?' Reporting irrelevant demographics in qualitative research," Qualitative Health Research, vol. 18, no. 3, pp. 299-300, 2008.
[2] J. Lynn and D. M. Adamson, "Living well at the end of life,” RAND Health, 2003, http://www.rand.org/pubs/white_ papers/WP137.html.

[3] D. J. Casarett and T. E. Quill, "I'm not ready for hospice': strategies for timely and effective hospice discussions," Annals of Internal Medicine, vol. 146, no. 6, pp. 443-449, 2007.

[4] E. A. Coleman, "Falling through the cracks: challenges and opportunities for improving transitional care for persons with continuous complex care needs," Journal of the American Geriatrics Society, vol. 51, no. 4, pp. 549-555, 2003.

[5] E. A. Coleman and C. Boult, "Improving the quality of transitional care for persons with complex care needs," Journal of the American Geriatrics Society, vol. 51, no. 4, pp. 556-557, 2003.

[6] A. Shih, K. Davis, S. Schoenbaum, A. Gauthier, R. Nuzum, and D. McCarthy, "Organizing the U.S. Health Care Delivery System for High Performance," The Commonwealth Fund, August 2008.

[7] J. M. Morse, "Confusing categories and themes," Qualitative Health Research, vol. 18, no. 6, pp. 727-728, 2008.

[8] M. P. Davis, D. Walsh, S. B. Legrand, and R. Lagman, "End-oflife care: the death of palliative medicine?" Journal of Palliative Medicine, vol. 5, no. 6, pp. 813-814, 2002.

[9] B. R. Ferrell, "Late referrals to palliative care," Journal of Clinical Oncology, vol. 23, no. 12, pp. 2588-2589, 2005.

[10] D. E. Meier, "The development, status, and future of palliative care," in Palliative Care: Transforming the Care of Serious Illness, D. E. Meier, S. L. Isaacs, and R. G. Hughes, Eds., pp. 3-76, Jossey-Bass, San Francisco, Calif, USA, 2002.

[11] R. S. Morrison, "Health care system factors affecting end-oflife care," Journal of Palliative Medicine, vol. 8, no. 1, pp. S-79S-87, 2005.

[12] T. E. Quill, "In-hospital end-of-life services: is the cup $2 / 3$ empty or 1/3 full?" Medical Care, vol. 40, no. 1, pp. 4-6, 2002.

[13] M. Ersek and B. R. Ferrell, "Palliative care nursing education: opportunities for gerontological nurses," Journal of Gerontological Nursing, vol. 31, no. 7, pp. 45-51, 2005.

[14] B. A. Powers and N. M. Watson, "Meaning and practice of palliative care for nursing home residents with dementia at end of life," American Journal of Alzheimer's Disease and other Dementias, vol. 23, no. 4, pp. 319-325, 2008.

[15] D. B. Bekelman, E. Hutt, F. A. Masoudi, J. S. Kutner, and J. S, Rumsfeld, "Defining the role of palliative care in older adults with heart failure," International Journal of Cardiology, vol. 125, no. 2, pp. 183-190, 2008.

[16] S. J. Goodlin, P. J. Hauptman, R. Arnold et al., "Consensus statement: palliative and supportive care in advanced heart failure," Journal of Cardiac Failure, vol. 10, no. 3, pp. 200-209, 2004.

[17] K. L. Grady, K. Dracup, G. Kennedy et al., “Team management of patients with heart failure: a statement for Healthcare Professionals from the Cardiovascular Nursing Council of the American Heart Association," Circulation, vol. 102, no. 19, pp. 2443-2456, 2000.

[18] S. J. Goodlin, R. Trupp, P. Bernhardt, K. L. Grady, and K. Dracup, "Development and evaluation of the "Advanced Heart Failure Clinical Competence Survey": a tool to assess knowledge of heart failure care and self-assessed competence," Patient Education and Counseling, vol. 67, no. 1-2, pp. 3-10, 2007.

[19] J. E. Hupcey, J. Penrod, and J. Fogg, "Heart failure and palliative care: implications in practice," Journal of Palliative Medicine, vol. 12, no. 6, pp. 531-536, 2009. 
[20] S. A. Norton, V. P. Tilden, S. W. Tolle, C. A. Nelson, and S. T. Eggman, "Life support withdrawal: communication and conflict," American Journal of Critical Care, vol. 12, no. 6, pp. 548-555, 2003.

[21] S. A. Norton and K. A. Talerico, "Facilitating end-of-life decision-making: strategies for communicating and assessing," Journal of Gerontological Nursing, vol. 26, no. 9, pp. 6-13, 2000.

[22] S. H. Ahmedzai and D. Walsh, "Palliative medicine and modern cancer care," Seminars in Oncology, vol. 27, no. 1, pp. $1-6,2000$.

[23] T. Quill, S. Norton, M. Shah, Y. Lam, C. Fridd, and M. Buckley, "What is most important for you to achieve?: an analysis of patient responses when receiving palliative care consultation," Journal of Palliative Medicine, vol. 9, no. 2, pp. 382-388, 2006.

[24] I. G. Finlay, I. J. Higginson, D. M. Goodwin et al., "Palliative care in hospital, hospice, at home: results from a systematic review," Annals of Oncology, vol. 13, supplement 4, pp. 257264, 2002.

[25] I. J. Higginson, I. Finlay, D. M. Goodwin et al., "Do hospitalbased palliative teams improve care for patients or families at the end of life?" Journal of Pain and Symptom Management, vol. 23, no. 2, pp. 96-106, 2002.

[26] D. Casarett, A. Pickard, F. A. Bailey et al., "Do palliative consultations improve patient outcomes?" Journal of the American Geriatrics Society, vol. 56, no. 4, pp. 593-599, 2008.

[27] E. L. Ciemins, L. Blum, M. Nunley, A. Lasher, and J. M. Newman, "The economic and clinical impact of an inpatient palliative care consultation service: a multifaceted approach," Journal of Palliative Medicine, vol. 10, no. 6, pp. 1347-1355, 2007.

[28] National Consensus Project for Quality Palliative Care, "Clinical practice guidelines for quality palliative care," 2 nd edition, 2009, http://www.nationalconsensusproject.org/whatispc.asp. 


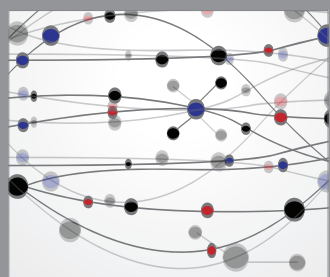

The Scientific World Journal
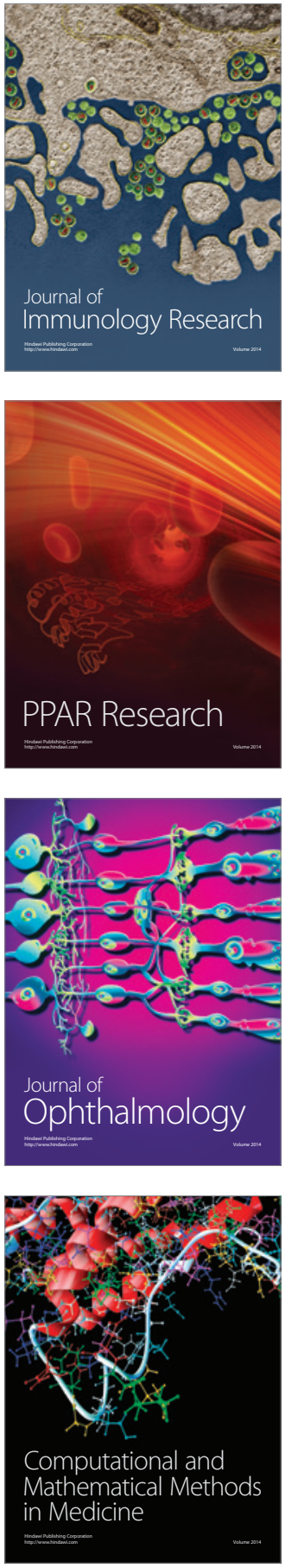

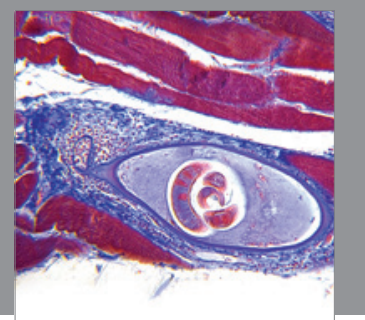

Gastroenterology

Research and Practice
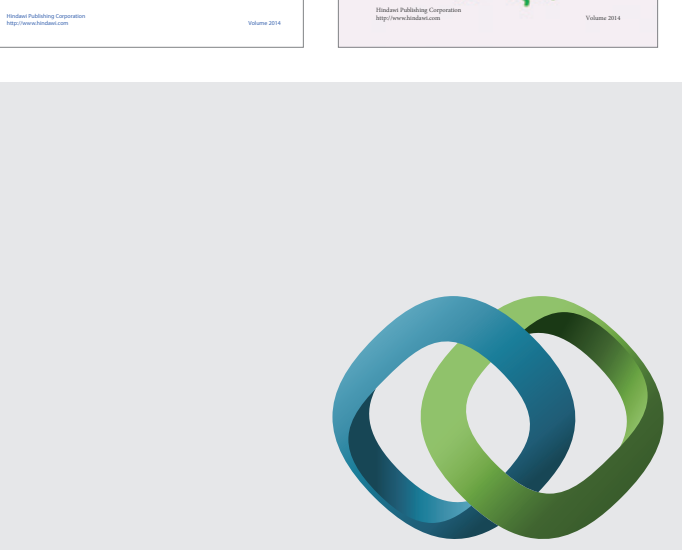

\section{Hindawi}

Submit your manuscripts at

http://www.hindawi.com
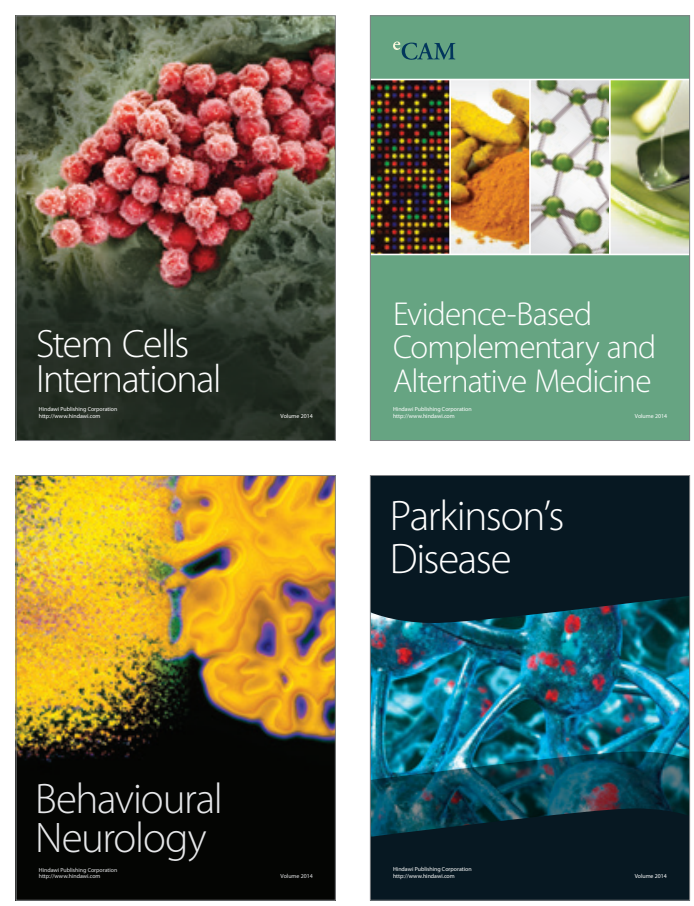

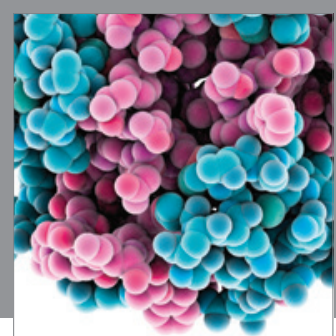

Journal of
Diabetes Research

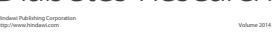

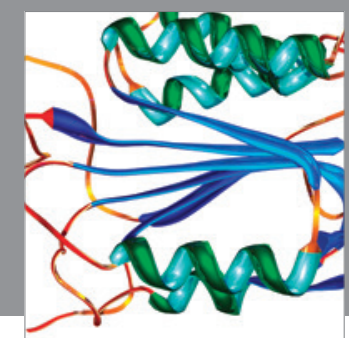

Disease Markers
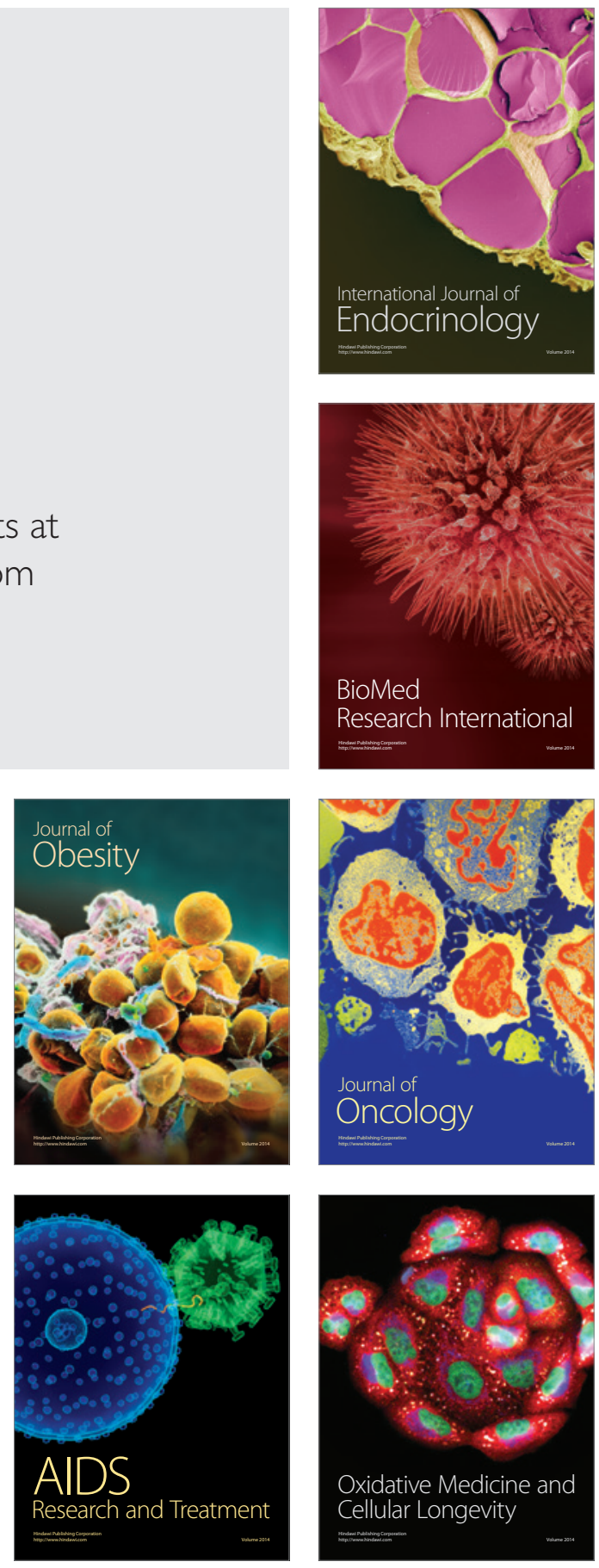